NOTE ON MIXED LINEAR INTEGRAL EQUATIONS.

BY DR. W. A. HURWITZ.

(Read before the American Mathematical Society, September 12, 1911.)

$\mathrm{Br}$ a mixed integral equation is here understood an equation in which an unknown function may be involved under several integral signs operating over regions of different numbers of dimensions, and in which also the values of the unknown function at isolated given points may occur. The highest number of dimensions of any region of integration may be termed the order of the equation; an equation entirely free from integral signs may also be regarded as a mixed integral equation of order zero. An equation in which regions of integration of different numbers of dimensions do not occur may be called pure; equations of this sort constitute the type usually studied.

The object of the present paper is to give some indication of the extent to which mixed (non-pure) linear integral equations may be expected to follow certain laws which have seemed to govern linear equations in general (e. g., pure integral equations and systems of algebraic equations). It is found that the analogy is not complete; the principal result, however, follows the lines of the usual theories. It is stated, for the case of equations of the first order, in the following

Theorem: Let $f(x), k_{1}(x), k_{2}(x), \cdots, k_{m}(x)$ be continuous fuctions of $x$ in the interval

$$
R_{1}: \quad a \leqq x \leqq b,
$$

and $K(x, y)$ a continuous function of $x$ and $y$ in the region

$$
R_{2}: \quad a \leqq x \leqq b, \quad a \leqq y \leqq b ;
$$

let $x_{1}, x_{2}, \cdots, x_{m}$ be distinct points of $R_{1}$. Then if the homogeneous mixed linear integral equation

$$
u(x)=\sum_{i=1}^{m} k_{i}(x) u\left(x_{i}\right)+\int_{a}^{b} K(x, s) u(s) d s
$$

has no continuous solution in $R_{1}$, other than $u(x)=0$, the nonhomogeneous mixed linear integral equation

$$
u(x)=f(x)+\sum_{i=1}^{m} k_{i}(x) u\left(x_{i}\right)+\int_{a}^{b} K(x, s) u(s) d s
$$

has one and only one continuous solution in $R_{1}$. 
To prove this, let us form the two normal orthogonal sets of principal solutions for the kernel $K(x, y)$

$$
\varphi_{1}(x), \varphi_{2}(x), \cdots, \varphi_{n}(x) ; \quad \psi_{1}(x), \psi_{2}(x), \cdots, \psi_{n}(x) ;
$$

these satisfy the following conditions:

$$
\begin{gathered}
\varphi_{j}(x)=\int_{a}^{b} K(x, s) \varphi_{j}(s) d s ; \quad \psi_{j}(x)=\int_{a}^{b} \psi_{j}(s) K(s, x) d s \\
(j=1,2, \cdots, n) ; \\
\int_{a}^{b}\left[\varphi_{j}(x)\right]^{2} d x=\int_{a}^{b}\left[\psi_{j}(x)\right]^{2} d x=1 ; \int_{a}^{b} \varphi_{i}(x) \varphi_{j}(x) d x= \\
\int_{a}^{b} \psi_{i}(x) \psi_{j}(x) d x=0 \quad(i \neq j ; i, j=1,2, \cdots, n) .
\end{gathered}
$$

The equations

$$
\begin{array}{r}
u_{0}(x)=f(x)-\sum_{j=1}^{n} \psi_{j}(x) \int_{a}^{b} \psi_{j}(s) f(s) d s+\int_{a}^{b} K(x, s) u_{0}(s) d s, \\
u_{i}(x)=k_{i}(x)-\sum_{j=1}^{n} \psi_{j}(x) \int_{a}^{b} \psi_{j}(s) k_{i}(s) d s+\int_{a}^{b} K(x, s) u_{i}(s) d s \\
(i=1,2, \cdots m)
\end{array}
$$

possess continuous solutions, since in each of them the known function outside the integral sign is orthogonal to $\psi_{1}(x)$, $\psi_{2}(x), \cdots, \psi_{n}(x)$. Form the following two systems of $m+n$ linear algebraic equations in $m+n$ unknowns $A_{1}, A_{2}, \cdots, A_{m}$; $B_{1}, B_{2}, \cdots, B_{n}$ :

$$
\begin{aligned}
&\left(\mathrm{I}_{1}\right) A_{p}-\sum_{i=1}^{m} A_{i} u_{i}\left(x_{p}\right)-\sum_{j=1}^{n} B_{j} \varphi_{j}\left(x_{p}\right)=0(p=1,2, \cdots, m), \\
&-\sum_{i=1}^{m} A_{i} \int_{a}^{b} \psi_{q}(s) k_{i}(s) d s=0(q=1,2, \cdots, n) ; \\
&\left(\mathrm{I}_{2}\right) \quad(p=1,2, \cdots, m) \\
&\left(\mathrm{II}_{1}\right) \mathrm{A}_{p}-\sum_{i=1}^{m} A_{i} u_{i}\left(x_{p}\right)-\sum_{j=1}^{n} B_{j} \varphi_{j}\left(x_{p}\right)=u_{0}\left(x_{p}\right) \\
&-\sum_{i=1}^{m} A_{i} \int_{a}^{b} \psi_{q}(s) k_{i}(s) d s=\int_{a}^{b} \psi_{q}(s) f(s) d s \\
&(q=1,2, \cdots, n) .
\end{aligned}
$$


Either system (I) possesses a set of solutions $A_{1}, A_{2}, \cdots, A_{m}$; $B_{1}, B_{2}, \cdots, B_{n}$ not all zero, or else system (II) possesses a set of solutions.*

Under the second of these alternatives, the continuous function

$$
u(x)=u_{0}(x)+\sum_{i=1}^{m} A_{i} u_{i}(x)+\sum_{j=1}^{n} B_{j} \varphi_{j}(x),
$$

defined in terms of the set of solutions of (II), is a solution of (2), as may be verified by direct substitution in (2), with the aid of (II). A second solution of (2) is impossible if (1) has no solutions not identically zero, since the difference of two solutions of (2) is evidently a solution of (1).

Under the first alternative, the continuous function

$$
u(x)=\sum_{i=1}^{m} A_{i} u_{i}(x)+\sum_{j=1}^{n} B_{j} \varphi_{j}(x),
$$

defined in terms of the set of solutions (not all zero) of (I), is similarly a solution of (1). Finally, this solution $u(x)$ is not identically zero; for if $u(x)=0$, then in particular $u\left(x_{p}\right)=0$ $(p=1,2, \cdots, m)$, so that (by substituting the values $x_{p}$ for $x$ in the definition of $u(x)$ and making use of $\left(\mathrm{I}_{1}\right)$ )

hence

$$
A_{p}=0 \quad(p=1,2, \cdots, m) ;
$$

$$
0=u(x)=\sum_{j=1}^{n} B_{j} \varphi_{j}(x),
$$

and in virtue of the normal orthogonality of $\varphi_{1}(x), \varphi_{2}(x)$, $\cdots, \varphi_{n}(x)$,

$$
B_{j}=0 \quad(j=1,2, \cdots, n),
$$

in contradiction to the fact that some one of $A_{1}, A_{2}, \cdots, A_{m}$; $B_{1}, B_{2}, \cdots, B_{n}$ is not zero.

The theorem stated above has thus been justified. With this theorem, however, the exactness of the analogy to the ordinary theories of linear equations seems to end. The more detailed study of the mixed linear integral equation is reserved for a later communication; an indication of the differences which present themselves is furnished by the

* The fact that these two states of affairs are not mutually exclusive is unessential. 
following remark, which is stated without proof. The rôle of the adjoint homogeneous equation in the ordinary theories* is here taken by a pure homogeneous linear integral equation together with linear integral auxiliary conditions

$$
\begin{array}{r}
v(x)=\int_{a}^{b} v(s) K(s, x) d s ; \quad \int_{a}^{b} v(x) k_{i}(x) d x=0 \\
(i=1,2, \cdots, m) ;
\end{array}
$$

but the numbers of independent solutions of (1) and $\left(1^{\prime}\right)$ need not, as in the usual cases, be the same. For example, there can never be more (and will in general be fewer) than $n$ linearly independent continuous solutions of $\left(1^{\prime}\right)$, while there may under certain conditions be as many as $m+n$ linearly independent continuous solutions of (1).

Cornell University, December 22, 1911 .

\section{NOTE ON THE GRAPHICAL SOLUTIONS OF THE FUNDAMENTAL EQUATIONS IN THE SHORT METHODS OF DETERMINING ORBITS.}

BY PROFESSOR A. O. LEUSCHNER AND MR. B. A. BERNSTEIN.

(Read before the San Francisco Section of the American Mathematical Society, October 28, 1911.)

ThE chief difficulty in the determination of preliminary orbits of new comets or asteroids arises from the fact that the geocentric or heliocentric distance of the new body enters into the problem as an unknown quantity. In the older methods the heliocentric distance often is assumed to be unity as a first approximation in the determination of comet orbits. The average heliocentric distance of the known asteroids is generally chosen as a first approximation for new asteroids. The true distances are then evaluated by laborious approximations.

In the short methods proposed by Professor Leuschner it is possible to make a direct determination of the geocentric dis-

* E. g., for the pure linear integral equation, the equation with transposed kernel; for the system of linear algebraic equations, the system with transposed (or conjugate) matrix. 